\title{
AÇão de Adjuvantes na Absorção e Translocação de Glyphosate em Plantas de AGUAPÉ (Eichhornia crassipes) ${ }^{1}$
}

\author{
The effect of Adjuvants on Glyphosate Absorption and Translocation in Water Hyacinth \\ (Eichhornia crassipes)
}

\author{
MARTINS, D. ${ }^{2}$, CARBONARI, C.A. ${ }^{3}$, TERRA, M.A. ${ }^{4}$ e MARCHI, S.R. ${ }^{5}$
}

\begin{abstract}
RESUMO - Este estudo teve por objetivo avaliar a ação de adjuvantes na absorção e translocação de glyphosate em plantas de aguapé (Eichhornia crassipes). Os tratamentos foram dispostos em esquema fatorial $5 \times$ 8, sendo: cinco tratamentos com glyphosate (sem adjuvante e com os adjuvantes Aterbane a 0,5 e 0,25\% v/v e Silwet L77 a 0,1 e 0,05\% $\mathrm{v} / \mathrm{v}$ ), aplicados somente em uma folha de cada planta; e oito intervalos para lavagem ou corte das folhas $(2,4,6,8,12,24$ e 48 horas, além de um intervalo continuo sem lavagem ou corte). Foram realizadas avaliações visuais de controle aos 7, 14, 21 e 28 dias após a aplicação dos tratamentos. Os adjuvantes Aterbane a 0,5\% e Silwet a 0,1\% promoveram maior controle em períodos mais curtos sem a lavagem das folhas (duas e quatro horas), embora no final do estudo todos os adjuvantes tenham resultado em controle eficiente a partir de duas horas, assim como o glyphosate sem adjuvante. No estudo de translocação, independentemente do adjuvante testado, observou-se excelente controle após o período de seis horas sem o corte das folhas. Nos periodos de duas e quatro horas, observou-se controle insatisfatório independentemente do adjuvante testado: esse fato demonstra que o corte das folhas em periodos inferiores a seis horas após a aplicação não é suficiente para uma translocação eficiente e garantir o controle de plantas de aguapé.
\end{abstract}

Palavras-chave: planta aquática, planta daninha, tecnologia de aplicação.

\begin{abstract}
This study was carried out to evaluate the effects of adjuvants on glyphosate absorption and translocation in Eichhornia crassipes. The treatments were arranged in a $5 \times 8$ factorial scheme, being five with glyphosate (with no adjuvant and with the adjuvants Aterbane at 0.5 and $0.25 \% v / v$ and Silwet $L 77$ at 0.1 and $0.05 \% v / v)$ applied on a single leaf of each plant at 8 intervals to wash or cut the leaves $(2,4,6,8,12,24$, and 48 hours, plus a continuous interval without washing or cutting). Visual control evaluations were carried out at 7, 14, 21, and 28 days after treatment application. The absorption of $0.5 \%$ Aterbane and $0.1 \%$ Silwet promoted a higher control after shorter periods without leaf washing (2 and 4 hours).However, by the end of the study, all the adjuvants showed an excellent control after the period of 6 hours, as well as glyphosate without adjuvant. For the translocation study, no leaf cutting after six hours promoted an excellent control, regardless of the adjuvant tested. For the 2 and 4 hour periods, an unsatisfactory control was observed, regardless of the adjuvant tested, showing that leaf cutting during periods below 6 hours after application is not sufficient for an efficient translocation and to guarantee control of the aquatic plants.
\end{abstract}

Keywords: aquatic weeds, weeds, application technology.

Recebido para publicação em 7.1.2007 e na forma revisada em 18.2.2009.

2 Professor Livre Docente, Dep. de Produção Vegetal, FCA/UNESP, Caixa Postal 237, 18603-970 Botucatu-SP, <dmartins@fca.unesp.br>; ${ }^{3}$ Aluno de Pós-Graduação do Dep. de Produção Vegetal, FCA/UNESP, Caixa Postal 237, 18603-970 Botucatu-SP; ${ }_{4}^{4}$ Professor, Dr., Faculdade Guaraí, 7770-000 Guaraí-TO, <marceloterra-to@uol.com.br>; ${ }^{5}$ Professor, Dr., Faculdade de Agronomia/UFMT, 78550-000 Sinop-MT, <sidneimarchi.ufmt@gmail.com>.

Planta Daninha, Viçosa-MG, v. 27, n. 1, p. 155-163, 2009 


\section{INTRODUÇÃO}

Lagos e reservatórios são fundamentais para o fornecimento de água para diversos sistemas, entre eles o agrícola. No entanto, décadas de desmatamento indiscriminado e uso inadequado do solo causaram o carregamento de nutrientes para o leito daqueles corpos hídricos, além de dejetos urbanos e industriais, sendo esses os principais fatores responsáveis pelo grande desequilíbrio existente e que acelera o crescimento exagerado da vegetação aquática indesejável.

Entre as diversas plantas aquáticas, destaca-se a espécie Eichhornia crassipes, a qual se caracteriza por ser aquática flutuante livre, nativa da América do Sul, pertencente à família Pontederidaceae e que se reproduz sexuadamente por sementes, viáveis por pelo menos 15 anos no sedimento dos corpos d'água (Holm \& Yeo, 1980), e de forma assexuada por estolões. Atualmente encontra-se amplamente distribuída nas regiões tropicais e subtropicais. A espécie é caracterizada como uma das plantas daninhas mais agressivas do mundo, presente em reservatórios e lagos (Holm et al., 1991). Essas plantas apresentam enorme potencial de reprodução, podendo aumentar sua área de cobertura em $15 \%$ ao dia, dobrando-a a cada seis ou sete dias (Hoyer et al., 1996).

O controle químico tem-se mostrado a opção mais eficaz e viável no controle de plantas aquáticas. Cada herbicida possui recomendações específicas para aplicação, que devem ser seguidas para garantir a eficiência e o uso seguro do produto, sem que haja consequências à saúde humana e ao meio ambiente (FAO, 1995).

O herbicida glyphosate é um inibidor da EPSPS, com ação sistêmica, facilmente translocável e provoca a morte do sistema radicular e de estruturas reprodutivas de plantas perenes, como bulbos e tubérculos (Kruse et al., 2000), com grande potencial de uso em ambientes aquáticos. As diferentes formulações dos inibidores da EPSPS apresentam baixa toxicidade para mamíferos, pássaros, peixes e a maioria das bactérias (Kruse et al., 2000).

A utilização de adjuvantes aumenta a eficácia de glyphosate, pelo aumento da retenção de gotas na folha e da absorção foliar, aumentando a hidratação da cutícula e incrementando a difusão de glyphosate através desta (Shilling et al., 1990; Reddy \& Singh, 1992b; Ruiter et al., 1992; Kruse et al., 2000). Ainda, o uso de adjuvantes pode reduzir o período sem chuvas necessário para que o glyphosate seja absorvido (Reddy \& Singh, 1992a).

Antes de apresentar ação fitotóxica, o herbicida deve ser absorvido via apoplasto e/ou simplasto e alcançar o seu sítio de ação, geralmente localizado no interior de uma organela (Hess \& Falk, 1990). No entanto, após atingir a superfície foliar, o herbicida está sujeito a vários destinos: escorrer, ser lavado pela ocorrência de chuva, secar e formar substância amorfa, cristalizar após a evaporação do solvente ou, ainda, penetrar na cutícula e permanecer retido nela, não sendo translocado (Werlang et al., 2003). A consequência imediata disso é a menor absorção e, consequentemente, a menor eficiência do herbicida.

A adição de adjuvantes pode elevar a absorção, porém a concentração desses adjuvantes deve ser bem estudada, pois podem ocorrer reduções no controle em função de altas concentrações desses adjuvantes, reduzindo a translocação do glyphosate (Sherrick et al., 1986). A absorção do glyphosate pela planta não implica necessariamente um bom controle, pois este está diretamente relacionado com a quantidade translocada na planta, pelo fato de o produto ser sistêmico.

Dessa forma, o objetivo deste trabalho foi avaliar a ação de adjuvantes na absorção e translocação de glyphosate em plantas de E. crassipes.

\section{MATERIAL E MÉTODOS}

Os experimentos foram instalados e conduzidos em casa de vegetação à temperatura de $25^{\circ} \mathrm{C}$, no Núcleo de Pesquisas Avançadas em Matologia, pertencente à Universidade Estadual Paulista, Botucatu-SP, Brasil.

O experimento foi conduzido em delineamento inteiramente casualizado, com quatro repetições. Os tratamentos foram dispostos em esquema fatorial $5 \times 8$, sendo 
testados dois adjuvantes, Aterbane e Silwet L77, em duas concentrações $(0,25 \%$ e $0,5 \%$ $\mathrm{v} / \mathrm{v}$ para o Aterbane e $0,05 \%$ e $0,1 \%$ para o Silwet L77), adicionados à calda do herbicida glyphosate (Rodeo), na dose de $1.680 \mathrm{~g} \mathrm{ha}^{-1}$, além de um tratamento sem adição de adjuvante. Foram estudados também oito intervalos de tempo para a lavagem (absorção) ou corte (translocação) das folhas: 2, 4, $6,8,12,24$ e 48 horas após a aplicação do herbicida, além de um tratamento sem lavagem ou corte das folhas.

As plantas de aguapé foram cultivadas em caixas plásticas, com as dimensões de $15 \times 15 \times 12 \mathrm{~cm}$, contendo cerca de $2 \mathrm{~L}$ de água, previamente adubada. As plantas receberam a aplicação do herbicida somente em uma folha predeterminada, sendo esta a folha central e maior; o restante das folhas de cada planta foi envolvido por sacos plásticos, evitando que estas tivessem contato com o herbicida.

Após a aplicação dos herbicidas, os sacos plásticos foram retirados e as plantas retornaram para a casa de vegetação, onde aguardaram os respectivos intervalos de tempo propostos nos tratamentos. Após esses intervalos, as folhas das plantas de cada tratamento foram lavadas ou cortadas, determinando-se, respectivamente, pelas posteriores avaliações de controle, o tempo de absorção e translocação do herbicida glyphosate. As folhas foram lavadas por meio de chuva simulada de $20 \mathrm{~mm}$, em um sistema estacionário.

O herbicida glyphosate foi aplicado em pós-emergência, utilizando-se um pulverizador estacionário pressurizado por ar comprimido com pressão constante de 1,5 bar, em laboratório fechado; a barra de aplicação foi equipada com quatro pontas tipo jato plano Teejet XR11002, distanciadas $50 \mathrm{~cm}$ entre si, a qual se deslocou à velocidade de $1 \mathrm{~m} \mathrm{~s}^{-1}$. A aplicação dos tratamentos foi feita com temperatura de $24 \pm 1{ }^{\circ} \mathrm{C}$ e umidade relativa do ar de $75 \pm 1 \%$.

A área foliar média das plantas foi determinada, e as caldas herbicidas foram concentradas, de forma que a quantidade de herbicida distribuída em uma folha fosse semelhante à quantidade total depositada sobre uma planta inteira em pulverização com consumo de calda de $200 \mathrm{~L} \mathrm{ha}^{-1}$.
As avaliações de controle das plantas foram visuais, por meio de uma escala percentual de notas, na qual 0 (zero) correspondeu a nenhuma injúria demonstrada pela planta e 100 (cem), à morte das plantas. Os parâmetros utilizados para estabelecimento das notas foram: acúmulo de biomassa, inibição do crescimento, quantidade e uniformidade das injúrias e capacidade de rebrota das plantas. As avaliações foram realizadas aos $7,14,21$ e 28 dias após aplicação do herbicida (DAA).

Os dados foram analisados por meio da análise de variância, efetuada pelo teste $\mathrm{F}$, e as médias dos tratamentos foram comparadas pelo teste de Tukey a 5\% de probabilidade.

\section{RESULTADOS E DISCUSSÃO}

Observa-se que, independentemente do período de avaliação de controle das plantas, quando estas foram lavadas ou cortadas, houve interação significativa pelo teste $F$ entre os diferentes fatores analisados (Tabelas 1 a 8). Na Tabela 1 são apresentados os resultados do controle das plantas de aguapé aos 7 DAA quando submetidas à lavagem das folhas em diferentes periodos após a aplicação de glyphosate. Pode-se observar que, para o Aterbane nas duas concentrações testadas e para o tratamento sem adjuvante, o controle foi crescente conforme o aumento dos períodos até seis horas sem que as folhas fossem lavadas. Para o Silwet a $0,1 \%$ o controle foi crescente até o período de seis horas, e na concentração de $0,05 \%$ o controle foi crescente até oito horas. Após esses períodos não ocorreram mais diferenças no controle das plantas de aguapé. De maneira geral, os adjuvantes não interferiram no controle após o intervalo de seis horas, porém, para o intervalo de duas horas o Silwet a $0,1 \%$ e o Aterbane a $0,25 \%$ promoveram maior controle.

Aos 14 DAA (Tabela 2), as plantas submetidas ao glyphosate com Aterbane a 0,5\% apresentaram bom controle (acima de 90\%) após o intervalo de quatro horas, enquanto o tratamento sem adjuvante e Silwet a 0,1\% apresentaram também bom controle somente após o intervalo de 48 horas. O uso de 
Aterbane a $0,25 \%$ mostrou controle satisfatório apenas após 12 horas, e o Silwet, após 24 horas. Ressalta-se que após esses períodos não ocorreram mais diferenças no controle proporcionado pelos diferentes adjuvantes e concentrações. Esse fato demonstra uma absorção e/ou translocação mais rápida do glyphosate promovida pelo Aterbane a $0,5 \%$, que, após o período de quatro horas, absorveu quantidade maior do herbicida, promovendo melhor controle. Reddy \& Sing (1992b) relatam que o período sem chuva, para uma boa ação do herbicida glyphosate, pode ser reduzido pela adição de adjuvantes.

Aos 21 e 28 DAA (Tabelas 3 e 4, respectivamente), as plantas já apresentavam controle bastante eficiente, independentemente do período e do adjuvante (acima de 94,5\%); no entanto, o Silwet a 0,05\% promoveu controle menos satisfatório que os demais para os periodos até quatro horas, o que demonstra que os adjuvantes não beneficiaram o desempenho final do glyphosate, uma vez que nenhum deles mostrou resultados melhores do que o glyphosate sem adjuvante. Ressalta-se que Pedrinho Junior et al. (2002) observaram efeito positivo da adição de uréia ao glyphosate no controle de plantas daninhas no verão em situações sujeitas a chuvas após a aplicação, o que não foi ora verificado para plantas de aguapé.

As plantas de aguapé demonstraram ser bastante suscetiveis ao glyphosate, pois o controle foi efetivo aos 28 DAA mesmo com a ocorrência de chuva após duas horas da aplicação. Esse resultado pode ter sido influenciado pelas condições em que foi aplicado o herbicida, ou seja, em sala fechada, sem ocorrência de ventos, o que maximizou a deposição do produto sobre as plantas; ainda, a dose pode estar acima da dose mínima necessária para promover controle satisfatório dessa espécie.

Esse período crítico entre a aplicação do herbicida em pós-emergência e a ocorrência de chuva pode variar em função de diversos fatores, como: ingrediente ativo, dose empregada, tipo de formulação, adjuvantes, quantidade e intensidade da chuva e, ainda, espécie de planta daninha (Jakelaitis et al., 2001; Pedrinho Junior et al., 2002; Werlang et al., 2003).

Tabela 1 - Porcentagem de controle de E. crassipes, aos 7 DAA, em função de adjuvantes e períodos de lavagem das folhas após aplicação de glyphosate. Botucatu-SP, 2004

\begin{tabular}{|c|c|c|c|c|c|}
\hline \multirow{2}{*}{ Período } & \multicolumn{5}{|c|}{ Adjuvante } \\
\hline & Sem adjuvante & Aterbane $0,5 \%$ & Aterbane $0,25 \%$ & Silwet $0,1 \%$ & Silwet $0,05 \%$ \\
\hline 2 horas & $17,5 \mathrm{C} \mathrm{b}^{1 /}$ & $25,0 \mathrm{Cb}$ & $34,3 \mathrm{C} \mathrm{a}$ & $39,5 \mathrm{C} \mathrm{a}$ & $18,0 \mathrm{D} \mathrm{b}$ \\
\hline 4 horas & $53,8 \mathrm{~B} \mathrm{~cd}$ & $62,0 \mathrm{~B} \mathrm{ab}$ & $59,5 \mathrm{~B} \mathrm{ab}$ & $66,5 \mathrm{~B} \mathrm{a}$ & $47,8 \mathrm{C} \mathrm{d}$ \\
\hline 6 horas & $67,8 \mathrm{~A} \mathrm{a}$ & $69,0 \mathrm{BC} \mathrm{a}$ & $70,3 \mathrm{~A} \mathrm{a}$ & $69,0 \mathrm{AB}$ a & $63,5 \mathrm{~B}$ a \\
\hline 8 horas & $70,8 \mathrm{~A} \mathrm{a}$ & $71,8 \mathrm{~A} \mathrm{a}$ & $74,0 \mathrm{~A} \mathrm{a}$ & $70,3 \mathrm{AB}$ a & $71,5 \mathrm{AB}$ a \\
\hline 12 horas & $71,5 \mathrm{~A} \mathrm{a}$ & $75,0 \mathrm{~A} \mathrm{a}$ & $70,3 \mathrm{~A}$ a & $70,8 \mathrm{AB}$ a & $76,0 \mathrm{~A} \mathrm{a}$ \\
\hline 24 horas & $72,0 \mathrm{~A} \mathrm{a}$ & $76,5 \mathrm{~A} \mathrm{a}$ & $75,8 \mathrm{~A} \mathrm{a}$ & $77,0 \mathrm{~A}$ a & $75,8 \mathrm{~A} \mathrm{a}$ \\
\hline 48 horas & $75,8 \mathrm{~A} \mathrm{a}$ & $74,5 \mathrm{~A} \mathrm{a}$ & $75,0 \mathrm{~A} \mathrm{a}$ & $76,5 \mathrm{~A} \mathrm{a}$ & $78,5 \mathrm{~A} \mathrm{a}$ \\
\hline sem lavagem & $76,5 \mathrm{~A} \mathrm{a}$ & $77,8 \mathrm{~A} \mathrm{a}$ & $79,0 \mathrm{~A} \mathrm{a}$ & $78,0 \mathrm{~A} \mathrm{a}$ & $77,8 \mathrm{~A} \mathrm{a}$ \\
\hline Adjuvantes (A) & \multicolumn{5}{|c|}{$8,88 * *$} \\
\hline Períodos (P) & \multicolumn{5}{|c|}{$300,84 * *$} \\
\hline $\mathrm{A}^{*} \mathrm{P}$ & \multicolumn{5}{|c|}{$3,90 * *$} \\
\hline $\mathrm{CV}(\%)$ & \multicolumn{5}{|c|}{6,6} \\
\hline $\operatorname{DMS}(\mathrm{A})$ & \multicolumn{5}{|c|}{8,55} \\
\hline DMS (P) & \multicolumn{5}{|c|}{9,52} \\
\hline
\end{tabular}

1 Médias seguidas de mesma letra, maiúscula na coluna e minúscula na linha, não diferem entre si pelo teste de Tukey ( $\mathrm{p}<0,05$ ). 
Tabela 2 - Porcentagem de controle de E. crassipes, aos 14 DAA, em função de adjuvantes e períodos de lavagem das folhas após aplicação de glyphosate. Botucatu-SP, 2004

\begin{tabular}{|c|c|c|c|c|c|}
\hline \multirow{2}{*}{ Período } & \multicolumn{5}{|c|}{ Adjuvante } \\
\hline & Sem adjuvante & Aterbane $0,5 \%$ & Aterbane $0,25 \%$ & Silwet $0,1 \%$ & Silwet $0,05 \%$ \\
\hline 2 horas & $77,0 \mathrm{C} \mathrm{ab}^{\underline{1 /}}$ & $78,8 \mathrm{~B} \mathrm{ab}$ & $82,5 \mathrm{D}$ a & $75,5 \mathrm{D}$ ab & $72,3 \mathrm{E} \mathrm{b}$ \\
\hline 4 horas & $80,5 \mathrm{BC}$ bc & $93,0 \mathrm{~A} \mathrm{a}$ & $83,8 \mathrm{D} \mathrm{b}$ & $87,5 \mathrm{C} \mathrm{ab}$ & $75,8 \mathrm{DE} \mathrm{c}$ \\
\hline 6 horas & $85,3 \mathrm{BC} \mathrm{c}$ & $95,8 \mathrm{~A} \mathrm{a}$ & $87,3 \mathrm{CD}$ bc & $93,3 \mathrm{ABC}$ ab & $82,0 \mathrm{CD} \mathrm{c}$ \\
\hline 8 horas & $86,5 \mathrm{~B}$ ab & $94,3 \mathrm{~A}$ a & $87,3 \mathrm{CD}$ ab & $88,8 \mathrm{BC}$ ab & $86,3 \mathrm{BC}$ ab \\
\hline 12 horas & $86,8 \mathrm{~B} \mathrm{~b}$ & $96,5 \mathrm{~A} \mathrm{a}$ & $90,3 \mathrm{ABC}$ ab & $87,5 \mathrm{C} \mathrm{b}$ & 84,0 BCD B \\
\hline 24 horas & $88,5 \mathrm{AB}$ a & $91,3 \mathrm{~A} \mathrm{a}$ & $90,8 \mathrm{ABC}$ a & $88,0 \mathrm{C} \mathrm{a}$ & $91,3 \mathrm{AB}$ a \\
\hline 48 horas & $96,0 \mathrm{~A}$ a & $97,3 \mathrm{~A}$ a & $95,5 \mathrm{AB}$ a & $97,8 \mathrm{~A} \mathrm{a}$ & $97,8 \mathrm{~A}$ a \\
\hline sem lavagem & $96,5 \mathrm{~A} \mathrm{a}$ & $97,8 \mathrm{~A} \mathrm{a}$ & $96,5 \mathrm{~A} \mathrm{a}$ & $97,5 \mathrm{AB}$ a & $96,8 \mathrm{~A} \mathrm{a}$ \\
\hline Adjuvantes (A) & \multicolumn{5}{|c|}{$15,12 * *$} \\
\hline Períodos $(\mathrm{P})$ & \multicolumn{5}{|c|}{$50,81 * *$} \\
\hline $\mathrm{A}^{*} \mathrm{P}$ & \multicolumn{5}{|c|}{$2,31 * *$} \\
\hline $\mathrm{CV}(\%)$ & \multicolumn{5}{|c|}{4,5} \\
\hline DMS (A) & \multicolumn{5}{|c|}{7,91} \\
\hline DMS (P) & \multicolumn{5}{|c|}{8,81} \\
\hline
\end{tabular}

1/ Médias seguidas de mesma letra, maiúscula na coluna e minúscula na linha, não diferem entre si pelo teste de Tukey (p $<0,05$ ).

Tabela 3 - Porcentagem de controle de E. crassipes, aos 21 DAA, em função de adjuvantes e períodos de lavagem das folhas após aplicação de glyphosate. Botucatu-SP, 2004

\begin{tabular}{|c|c|c|c|c|c|}
\hline \multirow{2}{*}{ Período } & \multicolumn{5}{|c|}{ Adjuvante } \\
\hline & Sem adjuvante & Aterbane $0,5 \%$ & Aterbane $0,25 \%$ & Silwet $0,1 \%$ & Silwet $0,05 \%$ \\
\hline 2 horas & $98,8 \mathrm{~A} \mathrm{a}^{1 /}$ & $99,0 \mathrm{~A}$ a & $99,5 \mathrm{~A} \mathrm{a}$ & $97,8 \mathrm{~A} \mathrm{a}$ & $94,5 \mathrm{~B} \mathrm{~b}$ \\
\hline 4 horas & $99,0 \mathrm{~A} \mathrm{a}$ & $100,0 \mathrm{~A} \mathrm{a}$ & $99,3 \mathrm{~A}$ a & $99,3 \mathrm{~A}$ a & $96,3 \mathrm{~B} \mathrm{~b}$ \\
\hline 6 horas & $98,8 \mathrm{~A} \mathrm{a}$ & $100,0 \mathrm{~A} \mathrm{a}$ & $99,5 \mathrm{~A} \mathrm{a}$ & $100,0 \mathrm{~A}$ a & $99,5 \mathrm{~A} \mathrm{a}$ \\
\hline 8 horas & $99,5 \mathrm{~A}$ a & $100,0 \mathrm{~A} \mathrm{a}$ & $99,8 \mathrm{~A} \mathrm{a}$ & $100,0 \mathrm{~A} \mathrm{a}$ & $99,5 \mathrm{~A}$ a \\
\hline 12 horas & $99,5 \mathrm{~A} \mathrm{a}$ & $100,0 \mathrm{~A} \mathrm{a}$ & $100,0 \mathrm{~A} \mathrm{a}$ & $100,0 \mathrm{~A}$ a & $99,5 \mathrm{~A} \mathrm{a}$ \\
\hline 24 horas & $100,0 \mathrm{~A} \mathrm{a}$ & $100,0 \mathrm{~A} \mathrm{a}$ & $100,0 \mathrm{~A} \mathrm{a}$ & $100,0 \mathrm{~A} \mathrm{a}$ & $100,0 \mathrm{~A}$ a \\
\hline 48 horas & $100,0 \mathrm{~A} \mathrm{a}$ & $100,0 \mathrm{~A} \mathrm{a}$ & $100,0 \mathrm{~A} \mathrm{a}$ & $100,0 \mathrm{~A} \mathrm{a}$ & $100,0 \mathrm{~A} \mathrm{a}$ \\
\hline sem lavagem & $97,8 \mathrm{~A} \mathrm{a}$ & $100,0 \mathrm{~A} \mathrm{a}$ & $100,0 \mathrm{~A} \mathrm{a}$ & $100,0 \mathrm{~A} \mathrm{a}$ & $100,0 \mathrm{~A} \mathrm{a}$ \\
\hline Adjuvantes (A) & \multicolumn{5}{|c|}{$4,67 * *$} \\
\hline Períodos (P) & \multicolumn{5}{|c|}{$5,90 * *$} \\
\hline $\mathrm{A} * \mathrm{P}$ & \multicolumn{5}{|c|}{$1,79 *$} \\
\hline $\mathrm{CV}(\%)$ & \multicolumn{5}{|c|}{1,3} \\
\hline DMS (A) & \multicolumn{5}{|c|}{2,61} \\
\hline DMS (P) & \multicolumn{5}{|c|}{2,90} \\
\hline
\end{tabular}

1 Médias seguidas de mesma letra, maiúscula na coluna e minúscula na linha, não diferem entre si pelo teste de Tukey ( $\mathrm{p}<0,05$ ). 
São encontradas na literatura variações nesse período crítico para diferentes espécies de plantas daninhas: Bariuan et al. (1999) constataram que o glyphosate necessitou de um periodo de 72 horas para que apresentasse máxima eficácia no controle de Cyperus rotundus; Bryson (1988) observou que o glyphosate necessitou de um período de quatro horas sem a ocorrência de chuvas para que não houvesse redução em sua eficácia em Sorghum halepense; e Silva (1996), por sua vez, observou que necessidade de um periodo sem chuva maior que 16 horas para que houvesse o máximo efeito do glyphosate sobre Brachiaria decumbens. Esses resultados indicam que a espécie e/ou dose empregadas foram determinantes nos resultados obtidos.

Na Tabela 5 estão apresentados os resultados do controle das plantas de aguapé aos 7 DAA quando submetidas ao corte da folha em diferentes períodos após a aplicação de glyphosate. Observa-se que o controle foi crescente conforme o aumento dos períodos, sendo mais eficiente após o intervalo de 48 horas, demonstrando que o produto apresentou translocação lenta, necessitando de mais de 48 horas para chegar ao restante da planta. Observaram-se efeitos dos adjuvantes nos resultados nos períodos avaliados; os tratamentos com Aterbane apresentaram melhores resultados para os períodos de 8 e 12 horas, porém o Silwet proporcionou bom controle para o período de 24 horas.

Aos 14 DAA (Tabela 6), as plantas submetidas ao intervalo de 48 horas e sem corte da folha apresentavam controle satisfatório, sendo este mais eficiente que nos demais periodos. Nos períodos iniciais, até quatro horas, o controle chegou no máximo a $15 \%$, sendo este insatisfatório independentemente do adjuvante utilizado, mostrando novamente que o produto apresentou um tempo longo para se translocar da folha para o restante da planta.

Para os períodos de seis e oito horas, o Silwet e o Aterbane, nas duas concentrações, apresentaram bons resultados, enquanto o tratamento sem adjuvante mostrou bom resultado somente após 12 horas sem corte.

Tabela 4 - Porcentagem de controle de E. crassipes, aos 28 DAA, em função de adjuvantes e períodos de lavagem das folhas após aplicação de glyphosate. Botucatu-SP, 2004

\begin{tabular}{|c|c|c|c|c|c|}
\hline \multirow{2}{*}{ Período } & \multicolumn{5}{|c|}{ Adjuvante } \\
\hline & Sem adjuvante & Aterbane $0,5 \%$ & Aterbane $0,25 \%$ & Silwet $0,1 \%$ & Silwet $0,05 \%$ \\
\hline 2 horas & $99,8 \mathrm{~A} \mathrm{a}^{1 /}$ & $99,8 \mathrm{~A} \mathrm{a}$ & $99,8 \mathrm{~A} \mathrm{a}$ & $99,5 \mathrm{~A} \mathrm{a}$ & $97,5 \mathrm{C} \mathrm{b}$ \\
\hline 4 horas & $100,0 \mathrm{~A} \mathrm{a}$ & $100,0 \mathrm{~A} \mathrm{a}$ & $100,0 \mathrm{~A} \mathrm{a}$ & $100,0 \mathrm{~A} \mathrm{a}$ & $98,8 \mathrm{~B} \mathrm{~b}$ \\
\hline 6 horas & $100,0 \mathrm{~A} \mathrm{a}$ & $100,0 \mathrm{~A} \mathrm{a}$ & $100,0 \mathrm{~A} \mathrm{a}$ & $100,0 \mathrm{~A}$ a & $100,0 \mathrm{~A} \mathrm{a}$ \\
\hline 8 horas & $100,0 \mathrm{~A} \mathrm{a}$ & $100,0 \mathrm{~A} \mathrm{a}$ & $100,0 \mathrm{~A} \mathrm{a}$ & $100,0 \mathrm{~A} \mathrm{a}$ & $100,0 \mathrm{~A} \mathrm{a}$ \\
\hline 12 horas & $100,0 \mathrm{~A} \mathrm{a}$ & $100,0 \mathrm{~A} \mathrm{a}$ & $100,0 \mathrm{~A} \mathrm{a}$ & $100,0 \mathrm{~A}$ a & $100,0 \mathrm{~A} \mathrm{a}$ \\
\hline 24 horas & $100,0 \mathrm{~A} \mathrm{a}$ & $100,0 \mathrm{~A} \mathrm{a}$ & $100,0 \mathrm{~A} \mathrm{a}$ & $100,0 \mathrm{~A} \mathrm{a}$ & $100,0 \mathrm{~A} \mathrm{a}$ \\
\hline 48 horas & $100,0 \mathrm{~A} \mathrm{a}$ & $100,0 \mathrm{~A} \mathrm{a}$ & $100,0 \mathrm{~A} \mathrm{a}$ & $100,0 \mathrm{~A} \mathrm{a}$ & $100,0 \mathrm{~A} \mathrm{a}$ \\
\hline sem lavagem & $99,5 \mathrm{~A} \mathrm{a}$ & $100,0 \mathrm{~A} \mathrm{a}$ & $100,0 \mathrm{~A} \mathrm{a}$ & $100,0 \mathrm{~A} \mathrm{a}$ & $100,0 \mathrm{~A} \mathrm{a}$ \\
\hline Adjuvantes (A) & \multicolumn{5}{|c|}{$6,71 * *$} \\
\hline Períodos (P) & \multicolumn{5}{|c|}{$8,29 * *$} \\
\hline $\mathrm{A}^{*} \mathrm{P}$ & \multicolumn{5}{|c|}{$3,60 * *$} \\
\hline $\mathrm{CV}(\%)$ & \multicolumn{5}{|c|}{0,4} \\
\hline DMS (A) & \multicolumn{5}{|c|}{0,80} \\
\hline DMS (P) & \multicolumn{5}{|c|}{0,89} \\
\hline
\end{tabular}

${ }^{1 /}$ Médias seguidas de mesma letra, maiúscula na coluna e minúscula na linha, não diferem entre si pelo teste de Tukey $(\mathrm{p}<0,05)$. 
Tabela 5 - Porcentagem de controle de E. crassipes, aos 7 DAA, em função de adjuvantes e períodos de corte das folhas após aplicação de glyphosate. Botucatu-SP, 2004

\begin{tabular}{|c|c|c|c|c|c|}
\hline \multirow{2}{*}{ Período } & \multicolumn{5}{|c|}{ Adjuvante } \\
\hline & Sem adjuvante & Aterbane $0,5 \%$ & Aterbane $0,25 \%$ & Silwet $0,1 \%$ & Silwet $0,05 \%$ \\
\hline 2 horas & $2,8 \mathrm{E} \mathrm{a}^{1 /}$ & $5,0 \mathrm{E} \mathrm{a}$ & $2,8 \mathrm{E} \mathrm{a}$ & $2,9 \mathrm{D}$ a & $2,5 \mathrm{~F} \mathrm{a}$ \\
\hline 4 horas & $2,3 \mathrm{E} \mathrm{a}$ & $3,5 \mathrm{DE}$ a & $1,8 \mathrm{E} \mathrm{a}$ & $2,5 \mathrm{D}$ a & $2,3 \mathrm{~F} \mathrm{a}$ \\
\hline 6 horas & $9,5 \mathrm{D} \quad \mathrm{b}$ & $11,3 \mathrm{D} \mathrm{ab}$ & $16,3 \mathrm{D}$ a & $16,3 \mathrm{C} \mathrm{a}$ & $12,8 \mathrm{E} a b$ \\
\hline 8 horas & $24,5 \mathrm{C} \mathrm{bc}$ & $30,0 \mathrm{C} \mathrm{a}$ & $27,5 \mathrm{C} \mathrm{a}$ & $21,3 \mathrm{C} \mathrm{b}$ & $19,0 \mathrm{DE} \mathrm{b}$ \\
\hline 12 horas & $22 \mathrm{C} \mathrm{ab}$ & $27,0 \mathrm{C} \mathrm{a}$ & $25,0 \mathrm{C} \mathrm{ab}$ & $21,5 \mathrm{C} \mathrm{ab}$ & $21,0 \mathrm{D} \mathrm{b}$ \\
\hline 24 horas & $37,5 \mathrm{~B} \mathrm{~b}$ & $38,5 \mathrm{~B} \mathrm{c}$ & $36,0 \mathrm{~B} \mathrm{c}$ & $41,8 \mathrm{~B} \mathrm{ab}$ & $45,0 \mathrm{C} \mathrm{a}$ \\
\hline 48 horas & $74,5 \mathrm{~A} \mathrm{a}$ & $73,3 \mathrm{~A} \mathrm{a}$ & $73,5 \mathrm{~A} \mathrm{a}$ & $73,3 \mathrm{~A} \mathrm{a}$ & $70,8 \mathrm{~B} \mathrm{a}$ \\
\hline sem lavagem & $76,5 \mathrm{~A} \mathrm{a}$ & $77,8 \mathrm{~A} \mathrm{a}$ & $79,0 \mathrm{~A} \mathrm{a}$ & $78,0 \mathrm{~A} \mathrm{a}$ & $77,8 \mathrm{~A} \mathrm{a}$ \\
\hline Adjuvantes (A) & \multicolumn{5}{|c|}{$2,67 *$} \\
\hline Períodos (P) & \multicolumn{5}{|c|}{$1.947,33 * *$} \\
\hline $\mathrm{A} * \mathrm{P}$ & \multicolumn{5}{|c|}{$3,03 * *$} \\
\hline $\mathrm{CV}(\%)$ & \multicolumn{5}{|c|}{9,3} \\
\hline DMS (A) & \multicolumn{5}{|c|}{5,83} \\
\hline DMS (P) & \multicolumn{5}{|c|}{6,50} \\
\hline
\end{tabular}

1/ Médias seguidas de mesma letra, maiúscula na coluna e minúscula na linha, não diferem entre si pelo teste de Tukey $(\mathrm{p}<0,05)$.

Tabela 6 - Porcentagem de controle de E. crassipes, aos 14 DAA, em função de adjuvantes e períodos de corte das folhas após aplicação de glyphosate. Botucatu-SP, 2004

\begin{tabular}{|c|c|c|c|c|c|}
\hline \multirow{2}{*}{ Período } & \multicolumn{5}{|c|}{ Adjuvante } \\
\hline & Sem adjuvante & Aterbane $0,5 \%$ & Aterbane $0,25 \%$ & Silwet $0,1 \%$ & Silwet $0,05 \%$ \\
\hline 2 horas & $13,8 \mathrm{~F} \mathrm{a}^{1 /}$ & $13,5 \mathrm{C} \mathrm{a}$ & $7,8 \mathrm{E} \mathrm{a}$ & $8,5 \mathrm{D}$ a & $9,3 \mathrm{D}$ a \\
\hline 4 horas & $12,0 \mathrm{~F} \mathrm{ab}$ & $15,0 \mathrm{C} \mathrm{a}$ & $7,5 \mathrm{E} \mathrm{b}$ & $8,8 \mathrm{D}$ ab & $10,0 \mathrm{D} \mathrm{ab}$ \\
\hline 6 horas & $56,3 \mathrm{E} \mathrm{b}$ & $75,3 \mathrm{~B} \mathrm{a}$ & $68,3 \mathrm{D}$ a & $75,3 \mathrm{C} \mathrm{a}$ & $74,0 \mathrm{C} \mathrm{a}$ \\
\hline 8 horas & $72,8 \mathrm{D}$ a & $79,8 \mathrm{~B}$ a & $79,5 \mathrm{C} \mathrm{a}$ & $79,5 \mathrm{C} \mathrm{a}$ & $76,5 \mathrm{C} \mathrm{a}$ \\
\hline 12 horas & $85,3 \mathrm{C} \mathrm{a}$ & $81,5 \mathrm{~B}$ a & $85,3 \mathrm{BC} \mathrm{a}$ & $78,3 \mathrm{BC} \mathrm{a}$ & $81,0 \mathrm{C} \mathrm{a}$ \\
\hline 24 horas & $87,0 \mathrm{BC}$ ab & $82,3 \mathrm{~B} \mathrm{~b}$ & $88,5 \mathrm{~B}$ ab & $87,5 \mathrm{~B} \mathrm{ab}$ & $89,5 \mathrm{~B} \mathrm{a}$ \\
\hline 48 horas & $93,5 \mathrm{AB}$ a & $95,8 \mathrm{~A} \mathrm{a}$ & $91,3 \mathrm{AB}$ a & $96,3 \mathrm{~A}$ a & $97,8 \mathrm{~A} \mathrm{a}$ \\
\hline sem lavagem & $96,5 \mathrm{~A} \mathrm{a}$ & $97,5 \mathrm{~A} \mathrm{a}$ & $97,0 \mathrm{~A} \mathrm{a}$ & $97,5 \mathrm{~A} \mathrm{a}$ & $96,8 \mathrm{AB}$ a \\
\hline Adjuvantes (A) & \multicolumn{5}{|c|}{$22,38 * *$} \\
\hline Períodos $(\mathrm{P})$ & \multicolumn{5}{|c|}{$1.663,98 * *$} \\
\hline $\mathrm{A} * \mathrm{P}$ & \multicolumn{5}{|c|}{$17,28 * *$} \\
\hline $\mathrm{CV}(\%)$ & \multicolumn{5}{|c|}{5,5} \\
\hline DMS (A) & \multicolumn{5}{|c|}{7,24} \\
\hline DMS (P) & \multicolumn{5}{|c|}{8,06} \\
\hline
\end{tabular}

1 Médias seguidas de mesma letra, maiúscula na coluna e minúscula na linha, não diferem entre si pelo teste de Tukey ( $\mathrm{p}<0,05$ ). 
Tabela 7 - Porcentagem de controle de E. crassipes, aos 21 DAA, em função de adjuvantes e períodos de corte das folhas após aplicação de glyphosate. Botucatu-SP, 2004

\begin{tabular}{|c|c|c|c|c|c|}
\hline \multirow{2}{*}{ Período } & \multicolumn{5}{|c|}{ Adjuvante } \\
\hline & Sem adjuvante & Aterbane $0,5 \%$ & Aterbane $0,25 \%$ & Silwet $0,1 \%$ & Silwet $0,05 \%$ \\
\hline 2 horas & $23,3 \mathrm{D} \mathrm{b}^{1 / /}$ & $62,0 \mathrm{C} \mathrm{a}$ & $5,8 \mathrm{D} \mathrm{c}$ & $4,8 \mathrm{C} \mathrm{c}$ & $3,5 \mathrm{D} \mathrm{c}$ \\
\hline 4 horas & $26,3 \mathrm{D} \mathrm{bc}$ & $59,8 \mathrm{C} \mathrm{a}$ & $18,0 \mathrm{C} \mathrm{c}$ & $25,8 \mathrm{~B} \mathrm{bc}$ & $28,8 \mathrm{C} \mathrm{b}$ \\
\hline 6 horas & $81,5 \mathrm{C} \mathrm{b}$ & $90,0 \mathrm{~B} \mathrm{ab}$ & $87,5 \mathrm{~B}$ ab & $91,3 \mathrm{~A} \mathrm{a}$ & $91,3 \mathrm{~B} \mathrm{a}$ \\
\hline 8 horas & $88,3 \mathrm{BC}$ ab & $90,5 \mathrm{AB} a b$ & $97,0 \mathrm{AB}$ a & $92,0 \mathrm{~A} \mathrm{a}$ & $83,0 \mathrm{AB} b$ \\
\hline 12 horas & $98,0 \mathrm{AB}$ a & $96,0 \mathrm{AB}$ a & $95,0 \mathrm{AB}$ a & $92,5 \mathrm{~A} \mathrm{a}$ & $92,8 \mathrm{AB}$ a \\
\hline 24 horas & $94,5 \mathrm{AB}$ a & $97,8 \mathrm{AB}$ a & $95,5 \mathrm{AB}$ a & $97,3 \mathrm{~A}$ a & $99,8 \mathrm{~A} \mathrm{a}$ \\
\hline 48 horas & $100,0 \mathrm{~A} \mathrm{a}$ & $100,0 \mathrm{~A} \mathrm{a}$ & $98,5 \mathrm{~A} \mathrm{a}$ & $100,0 \mathrm{~A} \mathrm{a}$ & $100,0 \mathrm{~A} \mathrm{a}$ \\
\hline sem lavagem & $99,5 \mathrm{~A}$ a & $99,8 \mathrm{AB}$ a & $99,8 \mathrm{~A} \mathrm{a}$ & $100,0 \mathrm{~A} \mathrm{a}$ & $100,0 \mathrm{~A} \mathrm{a}$ \\
\hline Adjuvantes (A) & \multicolumn{5}{|c|}{$42,74 * *$} \\
\hline Períodos (P) & \multicolumn{5}{|c|}{$1.017,25 * *$} \\
\hline $\mathrm{A} * \mathrm{P}$ & \multicolumn{5}{|c|}{$19,74 * *$} \\
\hline $\mathrm{CV}(\%)$ & \multicolumn{5}{|c|}{6,2} \\
\hline DMS (A) & \multicolumn{5}{|c|}{8,91} \\
\hline DMS (P) & \multicolumn{5}{|c|}{9,92} \\
\hline
\end{tabular}

¹/ Médias seguidas de mesma letra, maiúscula na coluna e minúscula na linha, não diferem entre si pelo teste de Tukey ( $\mathrm{p}<0,05)$.

Tabela 8 - Porcentagem de controle de E. crassipes, aos 28 DAA, em função de adjuvantes e períodos de corte das folhas após aplicação de glyphosate. Botucatu-SP, 2004

\begin{tabular}{|c|c|c|c|c|c|}
\hline \multirow{2}{*}{ Período } & \multicolumn{5}{|c|}{ Adjuvante } \\
\hline & Sem adjuvante & Aterbane $0,5 \%$ & Aterbane $0,25 \%$ & Silwet $0,1 \%$ & Silwet $0,05 \%$ \\
\hline 2 horas & $6,5 \mathrm{C} \mathrm{b}^{-1 /}$ & $13,3 \mathrm{C} \mathrm{a}$ & $5,0 \mathrm{C} \mathrm{b}$ & $13,0 \mathrm{C} \mathrm{a}$ & $7,3 \mathrm{C} \mathrm{b}$ \\
\hline 4 horas & $27,0 \mathrm{~B}$ a & $18,8 \mathrm{~B} \mathrm{~b}$ & $18,0 \mathrm{~B} \mathrm{bc}$ & $18,8 \mathrm{~B} \mathrm{~b}$ & $14,5 \mathrm{~B} \mathrm{c}$ \\
\hline 6 horas & $99,5 \mathrm{~A} \mathrm{a}$ & $100,0 \mathrm{~A} \mathrm{a}$ & $99,5 \mathrm{~A} \mathrm{a}$ & $98,8 \mathrm{~A}$ a & $98,8 \mathrm{~A} \mathrm{a}$ \\
\hline 8 horas & $100,0 \mathrm{~A} \mathrm{a}$ & $100,0 \mathrm{~A} \mathrm{a}$ & $100,0 \mathrm{~A} \mathrm{a}$ & $100,0 \mathrm{~A} \mathrm{a}$ & $99,5 \mathrm{~A} \mathrm{a}$ \\
\hline 12 horas & $100,0 \mathrm{~A} \mathrm{a}$ & $100,0 \mathrm{~A} \mathrm{a}$ & $100,0 \mathrm{~A} \mathrm{a}$ & $100,0 \mathrm{~A} \mathrm{a}$ & $100,0 \mathrm{~A} \mathrm{a}$ \\
\hline 24 horas & $100,0 \mathrm{~A} \mathrm{a}$ & $100,0 \mathrm{~A} \mathrm{a}$ & $100,0 \mathrm{~A} \mathrm{a}$ & $100,0 \mathrm{~A}$ a & $100,0 \mathrm{~A} \mathrm{a}$ \\
\hline 48 horas & $100,0 \mathrm{~A} \mathrm{a}$ & $100,0 \mathrm{~A} \mathrm{a}$ & $100,0 \mathrm{~A} \mathrm{a}$ & $100,0 \mathrm{~A}$ a & $100,0 \mathrm{~A} \mathrm{a}$ \\
\hline sem lavagem & $100,0 \mathrm{~A}$ a & $100,0 \mathrm{~A} \mathrm{a}$ & $100,0 \mathrm{~A} \mathrm{a}$ & $100,0 \mathrm{~A} \mathrm{a}$ & $100,0 \mathrm{~A} \mathrm{a}$ \\
\hline Adjuvantes (A) & \multicolumn{5}{|c|}{$4,51 * *$} \\
\hline Períodos (P) & \multicolumn{5}{|c|}{$8.113,17 * *$} \\
\hline $\mathrm{A}^{*} \mathrm{P}$ & \multicolumn{5}{|c|}{$4,68 * *$} \\
\hline $\mathrm{CV}(\%)$ & \multicolumn{5}{|c|}{2,5} \\
\hline $\operatorname{DMS}(\mathrm{A})$ & \multicolumn{5}{|c|}{3,87} \\
\hline DMS (P) & \multicolumn{5}{|c|}{4,31} \\
\hline
\end{tabular}

${ }^{1 /}$ Médias seguidas de mesma letra, maiúscula na coluna e minúscula na linha, não diferem entre si pelo teste de Tukey ( $\mathrm{p}<0,05$ ). 
O melhor controle proporcionado pelos adjuvantes pode estar relacionado ao aumento da molhabilidade da superfície, à redução de tensão superficial e ângulo de contato da gota, além de aumentar a penetração cuticular (Sherrick, 1986; Kruse et al., 2000).

Aos 21 DAA, verificaram-se excelentes resultados de controle a partir do período de seis horas, sendo este mais efetivo após 12 horas para o Aterbane a 0,5 e 0,25\% e sem adjuvante e após 24 horas para o Silwet em ambas as concentrações (Tabela 7). Nos períodos até oito horas, observaram-se diferenças entre os adjuvantes; o Aterbane a $0,5 \%$ apresentou melhores resultados nos períodos até quatro horas. Nos períodos de seis e oito horas, os dois adjuvantes (Aterbane e Silwet), nas duas concentrações, passam a comportarse de forma semelhante (Tabela 7).

Aos 28 DAA (Tabela 8), pode-se observar que todos os tratamentos apresentaram excelente controle após o período de seis horas $(99,5 \%)$ sem o corte das folhas. Para os períodos de duas e quatro horas, observou-se controle insatisfatório, independentemente do adjuvante testado.

Com base nos resultados, pode-se afirmar que o corte das folhas de aguapé em periodos inferiores a seis horas após a aplicação do glyphosate não é suficiente para uma absorção e/ou translocação eficiente e garantir o controle de plantas.

\section{LITERATURA CITADA}

BARIUAN, J. V.; REDDY, K. N.; WILLS, G. Glyphosate injury, rainfastness, absortion, and translocation in purple nutsedge (Cyperus rotundus). Weed Technol., v. 13, n. 1, p. 112-119, 1999.

BRYSON, C. T. Effects or rainfall on foliar herbicides applied to seedling johnsongrass (Sorghum halepense). Weed Technol., v. 2, n. 2, p. 124-130, 1988.

FAO. Strategies for water hyacinth control. Report of a panel of experts meeting. Fort Lauderdale: 1995. p. 137-148.

HESS, F. D.; FALK, R. H. Herbicide deposition on the leaf surface. Weed Sci., v. 38, n. 3, p. 280-288, 1990.

HOLM, G. L. et al. The world's worst weeds. Malabar: Krieger Publishing Company, 1991. 609 p.
HOLM, L.; YEO, R. The biology control and utilization of aquatic weeds. Weeds Today, p. 7-13, 1980.

HOYER, M. V. et al. Florida freshwater plants: A handbook of commom aquatic plants in Florida lakes. Gainesville: University of Florida, Institute of Food and Agriculture Sciences, 1996. 256 p.

JAKELAITIS, A. et al. Controle de Digitaria horizontalis pelos herbicidas glyphosate, sulfosate e glifosate potássico submetidos a diferentes intervalos de chuva após a aplicação. Planta Daninha, v. 19, n. 2, p. 279-286, 2001

KRUSE, D. N. et al. Herbicidas inibidores da EPSPS: Revisão de literatura. R. Bras. Herbic., v. 1, n. 2, p. 139144, 2000.

PEDRINHO JUNIOR, A.F.F. et al. Influência da chuva na eficácia do glyphosate em mistura com adjuvantes na dessecação de plantas daninhas. Planta Daninha, v. 20, n. 2, p. 263-272, 2002.

REDDY, N. K.; SINGH, M. Organosilicone adjuvants increased the efficacy of glyphosate for control of weeds in citrus (Citrus spp.). Hortiscience, v. 27, n. 9, p. 1003-1005, 1992a.

REDDY, N. K.; SINGH, M. Organosilicone adjuvants effects on glyphosate efficacy and rainfastness. Weed Technol., v. 6, n. 3, p. 361-365, 1992 b.

RUITER, H.; MEINEN, E.; VERBEEK, A. M. Influence of the type and concentration of surfactant on glyphosate absorption; relevance of drop spreading and drying time. In: FOY, C. L. (Ed.). Adjuvants for agrochemicals. Boca Raton: CRC, 1992. 735p.

SHERRICK, S. T.; HOLT, H. A.; HESS, F. D. Effects of adjuvants and environment during plant development on glyphosate absorption and translocation in field bindweed (Convulvus arvensis). Weed Sci., v. 34, n. 6, p. 811-816, 1986.

SHILLING, D.G. et al. Influence of surfactants and additives on phytotoxicity of glyphosate to torpedograss. J. Aquatic Plant Manag., v. 28, n. 1, p. 23-27, 1990.

SILVA, M. A. S. Efeitos de períodos sem chuva, dosagens e volume de aplicação de glyphosate no controle de Brachiaria decumbens Stapf. Botucatu, 1996. 67 p. Dissertação (Mestrado em Agronomia) - Universidade Estadual Paulista, 1996.

WERLANG, R. C et al. Efeitos da chuva na eficiência de formulações e doses de glyphosate no controle de Brachiaria decumbens. Planta Daninha, v. 21, n. 1, p. 121-130, 2003. 